\title{
El ultrasonido pulmonar en cardiología: realidades y promesas
}

\section{Lung ultrasound in cardiology: realities and promises}

\author{
Rodrigo Gopar-Nieto', Gabriela P. Alanís-Estrada², Diestefano E. Ronquillo-Ramírez², \\ Jorge L. Vargas-Estrada², Alexandra Arias-Mendoza², Gustavo Rojas-Velasco ${ }^{3}$ y \\ Diego Araiza-Garaygordobil**
}

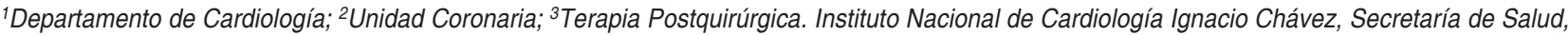
Ciudad de México, México

\section{Resumen}

El ultrasonido pulmonar es una herramienta de fácil reproducibilidad en pacientes estables y críticos que se utiliza para valorar el estado de congestión y comprobar la causa de la disnea. En este texto se revisan los fundamentos de la ecografía pulmonar, los protocolos de adquisición y su interpretación, así como la evidencia que sustenta su uso en el paciente cardiovascular críticamente enfermo y en el paciente estable. Esta herramienta debe emplearse como complemento de la exploración física regular para poder instituir un tratamiento oportuno en los pacientes con congestión pulmonar.

Palabras clave: Ultrasonido pulmonar. Insuficiencia cardíaca. Disnea.

\begin{abstract}
Lung ultrasound is an easily available, reproducible examination tool, both in ambulatory and critically-ill patients, which is used to evaluate congestion status and to differentiate the etiology of dyspnea. In this review, we explain lung ultrasound technique, acquisition protocols and their interpretation, as well as the evidence that shows its effectiveness among stable and critically-ill patients. Lung ultrasound should be used as an add-on to traditional physical examination in order to give an accurate diagnosis and a rapid treatment to patients with pulmonary congestion.
\end{abstract}

Key words: Lung ultrasound. Heart failure. Dyspnea.

\section{Correspondencia:}

*Diego Araiza-Garaygordobil

Juan Badiano, 1

Col. Belisario Domínguez Sección XVI, Del. Tlalpan

C.P. 14080 , Ciudad de México, México

E-mail: dargaray@gmail.com

1405-9940/@ 2019 Instituto Nacional de Cardiología Ignacio Chávez. Publicado por Permanyer. Este es un artículo open access bajo la licencia CC BY-NC-ND (http://creativecommons.org/licenses/by-nc-nd/4.0/).
Fecha de recepción: 04-04-2019

Fecha de aceptación: 04-06-2019
Disponible en internet: 05-08-2019 Arch Cardiol Mex. 2019;89(4):369-375

www.archivoscardiologia.com 


\section{Introducción}

El ultrasonido pulmonar (USP) es una técnica enfocada, rápida e inocua para el paciente y de fácil reproducción para el operador, y ha comenzado a formar parte de las herramientas del cardiólogo desde una década antes. A pesar de esto, aún dista de aplicarse de forma sistemática en todos los escenarios de la medicina cardiovascular, en particular debido a barreras ideológicas, como lo fueron las establecidas en textos clásicos de medicina interna de finales del siglo $X X^{1}$, en los cuales se aseguraba que el pulmón es un obstáculo para la imagen torácica. Empero, los trabajos de varios autores, en especial Lichtenstein, mostraron la utilidad de la ecografía pulmonar como herramienta en el tratamiento del paciente con condiciones críticas y con posterioridad se establecieron las bases para su uso en el paciente cardiovascular estable y descompensado 2 , a tal grado que guías como las de la Sociedad Europa de Cardiología recomiendan el uso del ultrasonido pulmonar en la insuficiencia cardíaca aguda con un grado de evidencia IIB $^{3}$.

Con la disponibilidad de ultrasonidos de bolsillo cada vez mayor, la ecografía pulmonar y de otros órganos debe aplicarse cada vez más en la práctica clínica diaria. El propósito de esta revisión es exponer los fundamentos de la ecografía pulmonar, los protocolos de adquisición y su interpretación, así como la evidencia que sustenta su uso en el paciente cardiovascular críticamente enfermo y en el sujeto estable. Por último, se muestran las lagunas de conocimiento que es necesario solventar mediante estudios de investigación para instituir esta técnica de manera más eficiente en la práctica diaria del cardiólogo.

\section{Fundamentos del USP}

El USP se basa en la observación de una serie de artefactos producidos por la interacción entre el ultrasonido y las interfaces pleural y alveolar y el aire de los alvéolos. Existen varias condiciones que pueden alterar esta interacción y producir efectos patológicos, entre ellos presencia de líquido, aire, edema alveolar, consolidación, que pueden identificarse como una serie de hallazgos ecográficos que proyectan al menos 10 signos que permiten el estudio ultrasonográfico del pulmón. En promedio, una revisión con USP puede realizarse en un minuto o menos, y la información obtenida puede orientar para descartar la presencia de derrame pleural o edema pulmonar o sugerir otras afecciones como neumotórax, neumonía o consolidación,
- EPOC, entre otras. La técnica de adquisición e interpretación puede estandarizarse en sesiones de un día o con enseñanza mediante módulos digitales disponibles en línea 4 .

\section{Técnica de adquisición}

En cuanto a la técnica de adquisición, hay que recalcar que el transductor debe colocarse de manera perpendicular a la pared torácica siguiendo la dirección de las costillas de manera oblicua. Los transductores de 3.5-5 MHz permiten una adecuada visualización de las estructuras subpleurales y cabe señalar que los transductores lineales detallan de mejor manera la pleura, mientras que los transductores cardíacos hacen posible visualizar mejor en términos de profundidad y alteraciones pulmonares ${ }^{4,5}$.

Una de las grandes ventajas es que la ventana acústica se encuentra casi siempre disponible, aun en casos en los que no es posible realizar un ecocardiograma transtorácico. En otras palabras, el ultrasonido pulmonar es factible en pacientes obesos, individuos críticamente enfermos, en posición supina, con ventilación mecánica invasiva y hemodinámicamente inestables. Para obtener vistas uniformes y fácilmente reproducibles se ha propuesto tener tres regiones simétricas por campo pulmonar, dos en la parte anterior y una posterolateral. Por último, es muy importante mencionar que el punto posterolateral sirve para corroborar la presencia de derrame pleural con una sensibilidad $>90 \%$ (Fig. 1$)^{6}$.

Resulta de particular interés para el cardiólogo detectar los cambios ocasionados por la acumulación de líquido en el espacio pleural o el parénquima pulmonar. En ambos casos, el USP tiene mejor rendimiento diagnóstico que la radiografía de tórax y sirve para iniciar el abordaje y diferenciar las causas de disnea, como neumonía, con una sensibilidad y especificidad de 89 y $94 \%$ respectivamente, en presencia de consolidación alveolar y un patrón difuso de líneas $B$ anteriores sin deslizamiento pleural, derrame pleural (muestra una mayor precisión en comparación con la radiografía portátil de tórax, $93 \%$ vs. $47 \%$ ), edema agudo pulmonar en presencia de líneas $B$ anteriores difusas con deslizamiento pulmonar (sensibilidad de $97 \%$ y especificidad de 94\%), así como enfermedad pulmonar obstructiva crónica, asma, tromboembolia pulmonar y neumotórax ${ }^{7}$.

Para la adquisición del USP se debe ajustar la profundidad del equipo de acuerdo con la complexión del paciente y se requiere menor profundidad en pacientes 


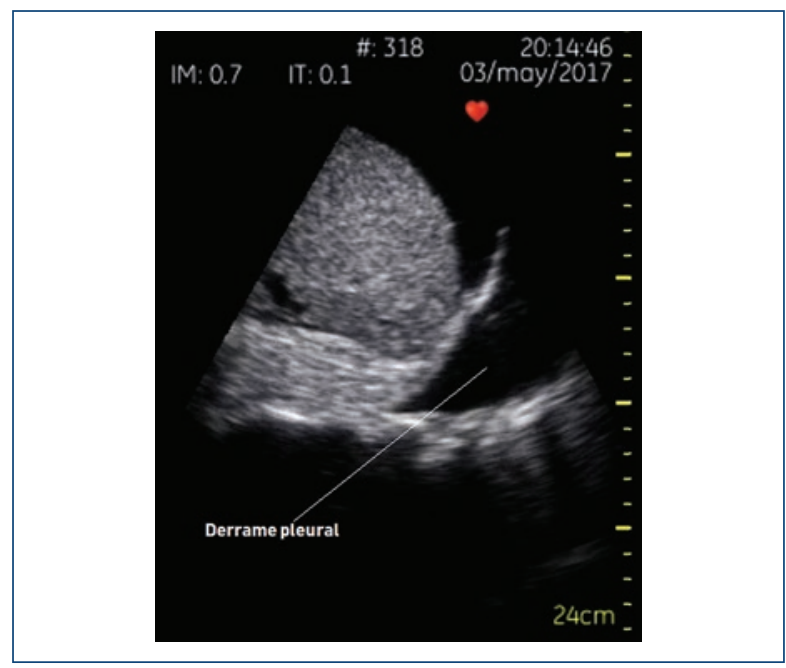

Figura 1. Ultrasonido pulmonar con imagen de derrame pleural.

delgados y mayor en los obesos, de tal modo que sea posible visualizar de manera adecuada la línea pleural. En la bibliografía mundial se han descrito al menos cinco tipos de protocolos para la adquisición de imágenes por medio de USP, los cuales incluyen imágenes de 28, 16, 8, 4 y 1 sitio $^{8}$. Se han comparado protocolos más extensos (28 sitios) con protocolos abreviados ( 3,4 y 8 sitios) en cuanto a su capacidad diagnóstica, y el área bajo la curva de los protocolos resumidos es mayor de 0.9 para detectar fenómenos patológicos comunes como congestión, considerando que la presencia de 3 o más líneas $B$ por campo, en dos o más campos por hemitórax, en ambos hemitórax, define la existencia de edema agudo pulmonar ${ }^{7}$, neumotórax o derrame, por lo que se ha propuesto la utilización de estos últimos para una visualización más rápida, sin la pérdida de datos relevantes (Fig. 2).

\section{Principales signos en USP}

La interacción normal del ultrasonido con el pulmón produce un artefacto conocido como líneas A (Fig. 3). Éstas son parte de un pulmón macroscópicamente normal y se producen por el eco de ambas pleuras (pleura parietal y visceral) que forman una "línea pleural". Ésta es visible a tan sólo unos centímetros debajo del transductor ultrasonográfico y, a su vez, muestra el movimiento de "deslizamiento" normal encontrado con la respiración (Fig. 4), el cual tiene una amplitud de 10 a $15 \mathrm{~mm}^{9}$. El eco que produce la línea pleural es a su vez visible y forma las líneas $A$, que constituyen otro hallazgo de pulmón normal y se encuentran a distancias equidistantes entre el transductor y la línea pleural, y pueden hallarse a razón de una, dos, tres o más en un pulmón saludable. La presencia de línea pleural con deslizamiento pleural y líneas $A$ indica un pulmón saludable. Los patrones anormales que se pueden encontrar ocurren en dos situaciones: a) cuando existe aire entre las capas pleurales, es decir, en el neumotórax, lo que da lugar a que el movimiento desaparezca, y b) cuando hay un exudado entre ambas capas, como en los procesos infecciosos, de manera tal que el movimiento disminuye o no existe.

El suceso fundamental que produce la insuficiencia cardíaca es el incremento de las presiones de llenado y el edema pulmonar cardiogénico. El edema alveolar cardiogénico, consecuencia de la elevación de las presiones diastólicas de la aurícula izquierda por diferentes motivos (disfunción ventricular izquierda sistólica, diastólica, o ambas, estenosis o regurgitación de la válvula mitral, obstrucción del tracto de salida del ventrículo izquierdo por diferentes motivos, isquemia aguda del ventrículo izquierdo, etc.) se relaciona con un incremento del grosor de la interfaz alveolocapilar. Este proceso, además de causar disnea, disminución de la oxigenación, tos y otros síntomas, provoca artefactos acústicos característicos, conocidos como líneas B o artefactos en cola de cometa por su semejanza con ese objeto astronómico.

Las líneas B tienen forma de cometa de orientación vertical, provienen de la línea pleural y se mueven de manera sincronizada con el deslizamiento pulmonar. Con bastante frecuencia se definen con forma de láser, borran a las líneas A y tienden a ser hiperecoicas (Fig. 5). Dentro de un mismo campo ultrasonográfico pueden identificarse una, dos o más líneas B; en ocasiones se reconocen individuales y diferenciables y se fusionan para formar lo que se conoce como un patrón "B", caracterizado por ocupar un porcentaje del área de rastreo y estar constituido por múltiples líneas $B$ fusionadas ${ }^{4,10}$. La cantidad de líneas $B$ es un parámetro de gravedad y se ha acompañado de un peor pronóstico, como se describe más adelante.

\section{USP e insuficiencia cardíaca y disnea}

La insuficiencia cardíaca, ya sea con fracción de expulsión disminuida o preservada, es una entidad fundamental en la patología cardiovascular y en la práctica diaria del cardiólogo. Por lo regular, el diagnóstico de insuficiencia cardíaca se ha basado en hallazgos de la exploración física y la historia clínica del paciente. Sin 


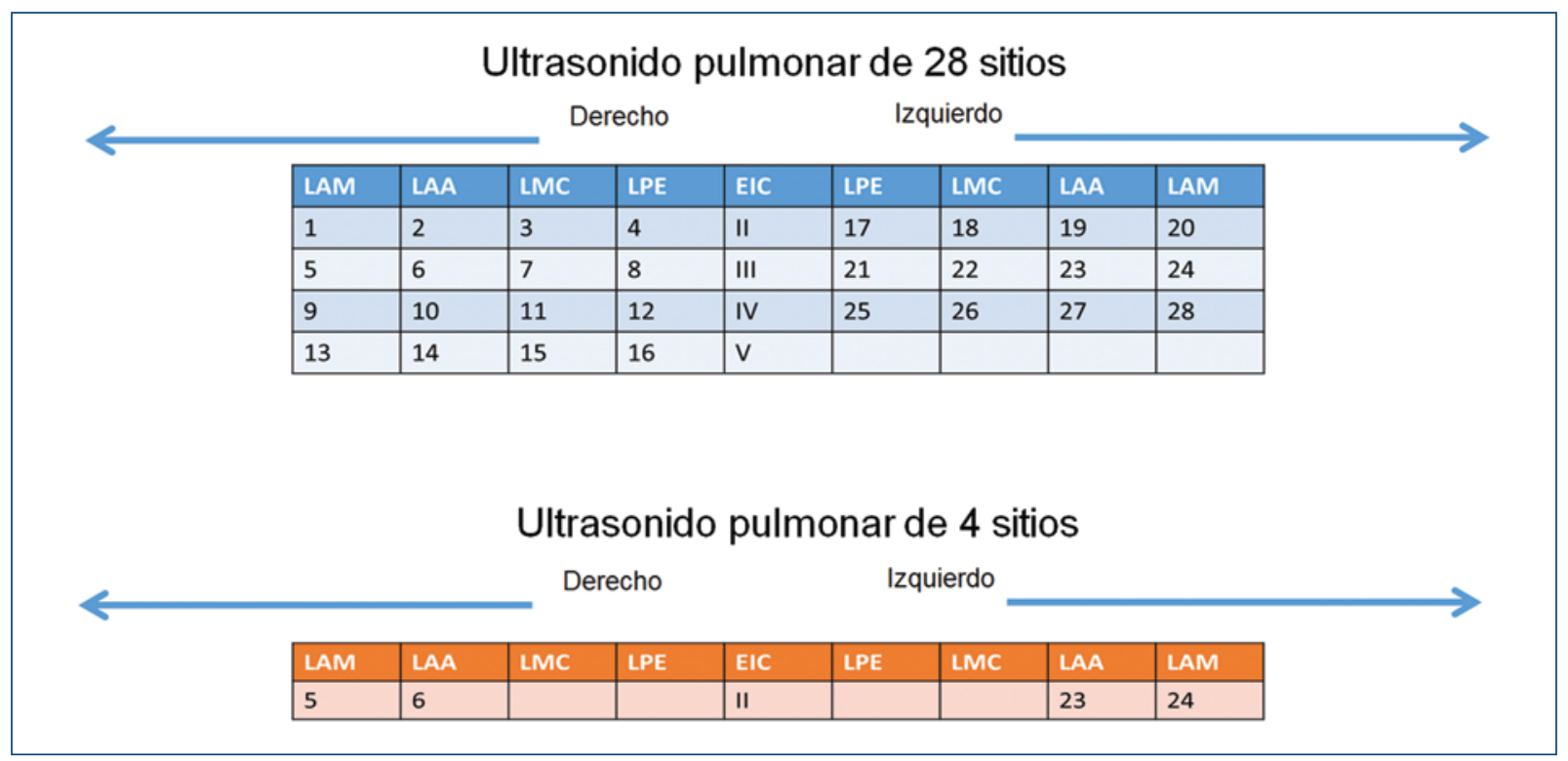

Figura 2. Comparación entre el protocolo de adquisición para ultrasonido pulmonar de 28 y 4 sitios.

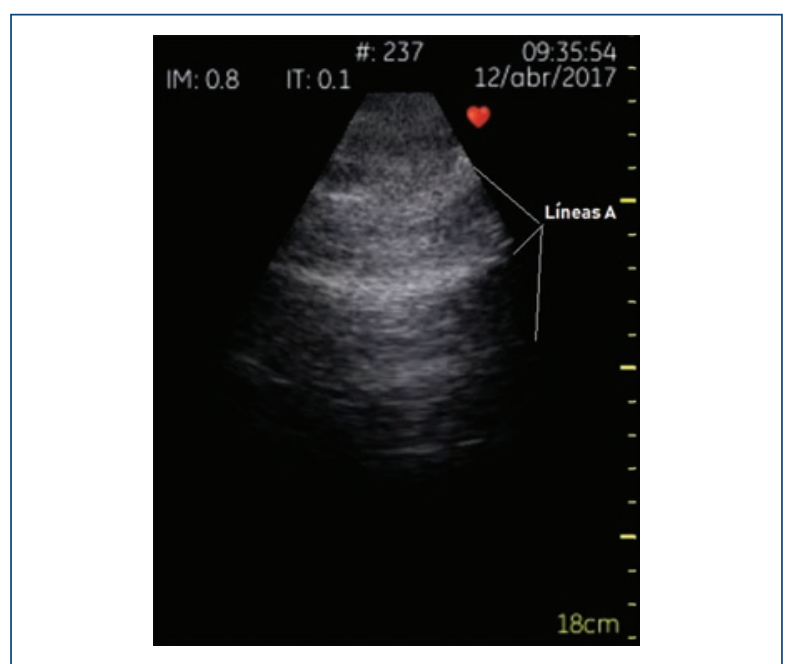

Figura 3. Líneas A visualizadas con ultrasonido pulmonar.

embargo, al incrementarse la prevalencia de la insuficiencia cardíaca, ha resultado evidente la necesidad de contar con herramientas clínicas con un poder diagnóstico y diferenciador superior al del estudio clínico (como el ecocardiograma y los péptidos natriuréticos cerebral y auricular).

EI USP puede considerarse una herramienta confiable para la evaluación de la congestión pulmonar en pacientes con insuficiencia cardíaca, ya que constituye una extensión de la exploración física y ayuda a diferenciar la hemodinámica de la congestión pulmonar.

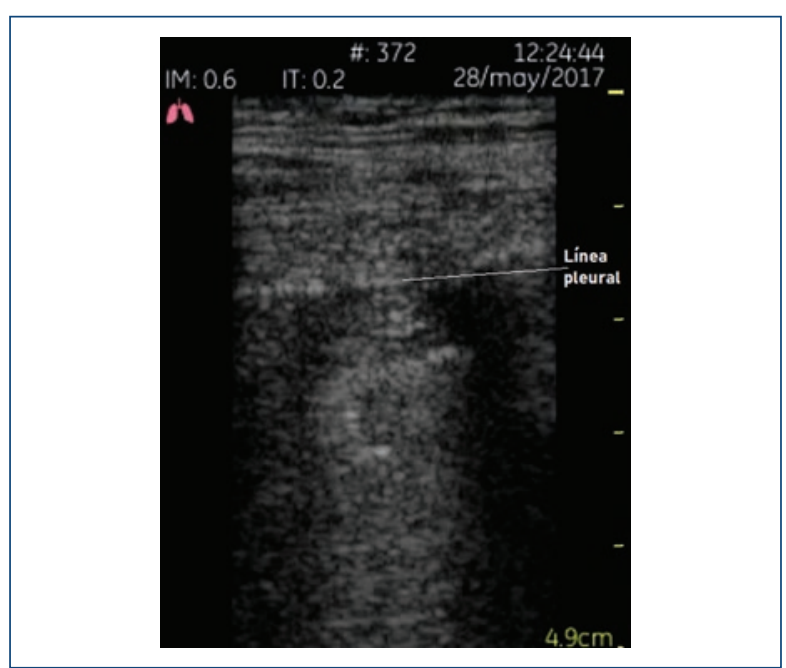

Figura 4. Línea pleural en ultrasonido pulmonar realizado con transductor lineal.

Dentro de los avances más recientes se ha intentado optimizar el tratamiento médico guiado por USP y se logró evidenciar una mejoría significativa en cuanto a la sensación de disnea (7.68 vs. 3.57, $p=0.007$, medido con la escala visual análoga de disnea) durante las primeras 12 horas de tratamiento; hay que mencionar además que la respuesta al tratamiento se relacionó con la sobrevida a largo plazo, que muestra una disminución de riesgo (HR) de 0.19 (intervalo de confianza $[\mathrm{IC}]$ del 95\%, 0.06-0.67, $p=0.010$ ) con respecto a la mortalidad por todas las causas ${ }^{11,12}$. 


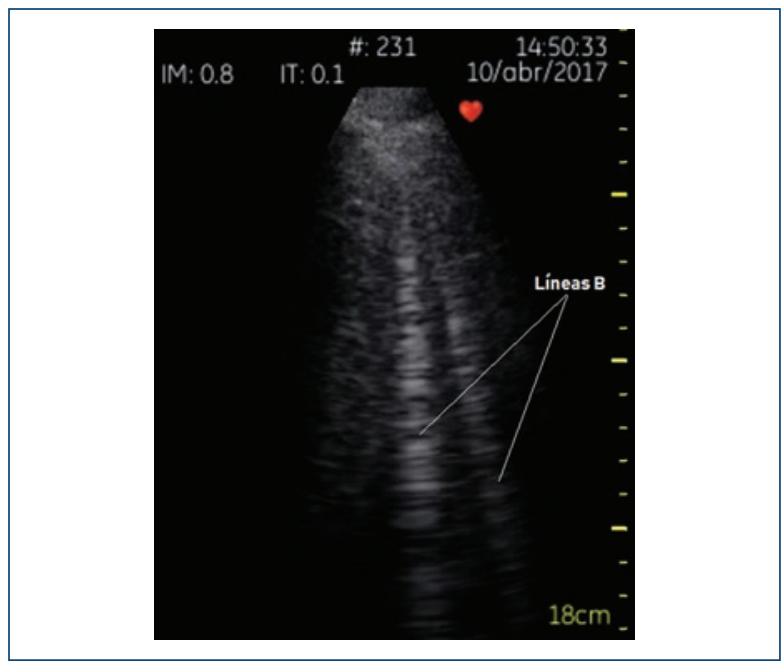

Figura 5. Múltiples líneas B con ultrasonido pulmonar.

Esta técnica es atractiva para la clínica ambulatoria porque utiliza tecnología simple, en la cual se pueden destacar los dispositivos de bolsillo, y es rápida de realizar e interpretar. Como posible implicación clínica, la farmacoterapia podría instituirse tan pronto como el paciente, aunque esté asintomático, muestre un aumento significativo del número de líneas $\mathrm{B}^{13}$. Por último, hay que mencionar que la congestión pulmonar también se ha estudiado en poblaciones especiales, como las personas con hemodiálisis, en las que se ha utilizado para detectar la congestión asintomática, y se ha encontrado que su presencia fue un predictor de muerte y episodios cardíacos; esto resalta la importancia de utilizar esta herramienta de manera temprana ${ }^{14}$.

Por otra parte, en el contexto de insuficiencia cardíaca crónica compensada, la evaluación de la congestión pulmonar se ha realizado por lo regular mediante la exploración física y la radiografía de tórax, pero en fecha más reciente el USP ha demostrado ser una herramienta sensible y semicuantitativa. Es en este aspecto en el que ha demostrado que la presencia de tres o más líneas $B$ se correlaciona con desenlaces cardiovasculares adversos, en particular con mayor riesgo de rehospitalizaciones y mortali$\mathrm{dad}^{15-17}$. Mientras tanto, en pacientes con insuficiencia cardíaca descompensada se ha utilizado el USP como predictor de nuevos ingresos hospitalarios tras la institución del tratamiento médico y antes del alta, tal y como lo demostraron Gargani, et al. En este estudio encontraron que la presencia $>50$ líneas $B$ en el momento del ingreso fue el mayor predictor de reingreso hospitalario por falla cardíaca durante el seguimiento con una sensibilidad de $71.4 \%$, especificidad de $69.8 \%$, valor predictivo negativo de $93.7 \%$, valor predictivo positivo de $27.8 \%$ (área bajo la curva de 0.71 , con intervalo de confianza del $95 \%, 0.58$ $0.85, p=0.011)^{18}$.

La disnea aguda es una causa frecuente de hospitalización y puede ocurrir en múltiples situaciones, entre ellas insuficiencia cardíaca descompensada aguda, exacerbación de la enfermedad pulmonar obstructiva crónica, estados de ansiedad y embolismo pulmonar. A pesar de la existencia de métodos paraclínicos modernos, la diferenciación entre las causas cardíacas y no cardíacas de disnea es todavía un problema clínico. El ultrasonido pulmonar ha adquirido relevancia, ya que se ha demostrado que la cantidad de cometas de ultrasonido pulmonar aumenta con el empeoramiento de la clase funcional de la New York Heart Association. Además de esto, se ha encontrado correlación con las líneas B de Kerley y la puntuación del agua pulmonar en la radiografía de tórax, el agua pulmonar extravascular medida de forma invasiva por el método de termodilución y la gravedad de la disfunción diastólica. Un metaanálisis reciente comparó la sensibilidad y precisión del ultrasonido pulmonar en comparación con la radiografía de tórax en pacientes con insuficiencia cardíaca aguda, incluidos seis estudios y un total de 1827 pacientes. El estudio notificó una sensibilidad para el USP de 0.88 (IC 95\%, 0.750.95) y especificidad de 0.90 (IC $95 \%, 0.88-0.92$ ), en comparación con una sensibilidad de 0.73 (IC 95\%, $0.70-0.76)$ para la radiografía de tórax, sin diferencias en cuanto a especificidad ${ }^{19}$. En otro estudio se comparó el rendimiento diagnóstico del USP contra los péptidos natriuréticos y se observó que las principales causas de la disnea cardiogénica fueron disfunción sistólica, disfunción diastólica aislada, valvulopatía grave y arritmias. Los valores de NT-proBNP se correlacionaron de manera positiva con el número de líneas $B(r=0.69, p<0001)$. El análisis ROC mostró que una concentración plasmática de NT-proBNP de $298 \mathrm{ng} / \mathrm{l}$ tuvo sensibilidad de $97 \%$ y especificidad del $92.6 \%$, mientras que la presencia de nueve líneas B tuvo sensibilidad de $73 \%$ y especificidad de $100 \%$. Este estudio comprobó que las líneas $B$ son un método simple y útil para el diagnóstico diferencial de la disnea aguda cardiogénica, respecto de la no cardiogénica, dado que proporcionan una imagen directa, morfológica y fácilmente distinguible de aumentos anormales en el agua de los pulmones ${ }^{20}$. 


\section{Perspectivas y limitaciones}

La clínica contemporánea necesita fundamentarse en la experiencia recopilada a través de los siglos y combinarla con los nuevos elementos que se generan gracias a los avances tecnológicos. Prueba de esto es la disponibilidad actual de los ultrasonidos de bolsillo, los cuales permiten tener una mejor valoración inicial del paciente. A pesar de esto no debe presuponerse que las nuevas tecnologías reemplazarán por completo a los métodos habituales, ya que la exploración física siempre tendrá un lugar esencial en la relación médico-paciente y será un punto de referencia del quehacer médico.

Dentro de las limitaciones del ultrasonido pulmonar debe señalarse que la única alteración que puede ocasionar dificultades para realizar un buen estudio es el enfisema subcutáneo grave. Por otra parte, dado que es un estudio relativamente novedoso y con aplicaciones en ramas muy diferentes de la medicina, puede suscitar confusión su aplicación porque es necesario definir valores de corte y metodologías estrictas para difundir su uso.

En cuanto a las nuevas tendencias puede afirmarse que hoy día existen varios estudios que intentan contestar las preguntas acerca del punto de corte de las líneas $B$ en estrés y reposo en pacientes con insuficiencia cardíaca o enfermedad coronaria, además de evaluar si guiar la terapéutica por medio de USP en pacientes con insuficiencia cardíaca aguda ${ }^{21}$ y cróni$\mathrm{ca}^{22}$ es igual o mejor que el tratamiento guiado por los hallazgos de la exploración física. En la población de este país sobresale el estudio CLUSTER-HF, que es el primer estudio latinoamericano que se encuentra en fase de inscripción, y busca establecer si el USP será útil para guiar el tratamiento médico en pacientes mexicanos con insuficiencia cardíaca (NCT03613779) ${ }^{22}$.

\section{Conclusiones}

El USP es una herramienta que complementa el gabinete diagnóstico del cardiólogo y ayuda a diferenciar, de manera rápida y eficaz, la sobrecarga pulmonar y la causa de disnea en pacientes críticos y ambulatorios. Una de las fortalezas de este estudio es su gran capacidad de reproducibilidad y el poco tiempo de adquisición, lo cual puede contribuir a seguir con su implementación como herramienta para el abordaje diagnóstico en todos los cardiólogos.

\section{Conflicto de intereses}

No existen conflictos de interés.

\section{Financiamiento}

Los autores no recibieron financiamiento alguno para la elaboración de este documento.

\section{Responsabilidades éticas}

Protección de personas y animales. Los autores declaran que para esta investigación no se han realizado experimentos en seres humanos ni en animales.

Confidencialidad de los datos. Los autores declaran que en este artículo no aparecen datos de pacientes.

Derecho a la privacidad y consentimiento informado. Los autores declaran que en este artículo no aparecen datos de pacientes.

\section{Bibliografía}

1. Longo D, Fauci AS, Kasper DL, Hauser SK, Jameson JL, Loscalzo J, editors. Harrison's principles of internal medicine. $18^{\text {th }}$ ed. New York: McGraw-Hill, 2011. Part 11, section 1, chapter 253.

2. Lichtenstein $D$, Mézière $G$, Biderman $P$, Gepner $A$, Barré $O$. The comet-tail artifact. An ultrasound sign of alveolar-interstitial syndrome. Am J Respir Crit Care Med 1997;156:1640-6.

3. Ponikowski P, Voors AA, Anker SD, Bueno H, Cleland JGF, Coats AJS, et al, ESC Scientific Document Group, 2016 ESC Guidelines for the diagnosis and treatment of acute and chronic heart failure: the task force for the diagnosis and treatment of acute and chronic heart failure of the European Society of Cardiology (ESC) Developed with the special contribution of the Heart Failure Association (HFA) of the ESC, European Heart Journal 2016;372129-2200, https://doi.org/10.1093/eurheartj/ehw128

4. Picano E, Scali MC, Ciampi Q, Lichtenstein D. Lung ultrasound for the cardiologist. J Am Coll Cardio IImg. 2018;11:1692-705.

5. Lichtenstein D. The probe. In: Lung Ultrasound in the Critically III - The BLUE-Protocol. Heidelberg, Germany: Springer, 2016; p 23-35.

6. Lichtenstein D, Lascols N, Méziére G, Gepner A. Ultrasound diagnosis of alveolar consolidation in the critically ill. Intensive Care Med. 2004; 30:276-81.

7. Lichtenstein $D$, Méziére $G$. Relevance of lung ultrasound in the diagnosis of acute respiratory failure: the BLUE protocol. Chest. 2008;134:117-25.

8. Scali MC, Zagatina A, Simova I, Zhuravskaya N, Ciampi Q, Paterni M, et al. B-lines with lung ultrasound: the optimal scan technique at rest and during stress. Ultrasound Med Biol. 2017;43:2558-2566.

9. Lichtenstein D. Lung ultrasound in the critically ill. Ann Intensive Care. 2014;4:1.

10. Xirouchaki N, Kondili E, Prinianakis G, Malliotakis P, Georgopoulos D. Impact of lung ultrasound on clinical decision making in critically ill patients. Intensive Care Med. 2014 Jan;40(1):57-65.

11. Öhman J, Harjola VP, Karjalainen P, Lassus J. Assessment of early treatment response by rapid cardiothoracic ultrasound in acute heart failure: Cardiac filling pressures, pulmonary congestion and mortality. Eur Heart J Acute Cardiovasc Care. 2018;7(4):311-320.

12. Öhman J, Harjola VP, Karjalainen P, Lassus J. Focused echocardiography and lung ultrasound protocol for guiding treatment in acute heart failure. ESC HeartFail. 2018;5(1):120-128.

13. Miglioranza MH, Gargani L, Sant'Anna RT, Rover MM, Martins VM, Mantovani $A$, et al. Lung ultrasound for the evaluation of pulmonary congestion in outpatients: a comparison with clinical assessment, natriuretic peptides, and echocardiography. JACC Cardiovasc Imaging. 2013; 6(11):1141-51.

14. Zoccali C, Torino C, Tripepi R, Tripepi G, D'Arrigo G, Postorino M, et al. Pulmonary congestion predicts cardiac events and mortality in ESRD. J Am Soc Nephrol. 2013 Mar;24(4):639-46.

15. Platz E, Lewis EF, Uno H, Peck J, Pivetta E, Merz AA, et al. Detection and prognostic value of pulmonary congestion by lung ultrasound in ambulatory heart failure patients. EurHeart J 2016;37(15):1244-51. 
16. Platz E, Merz AA, Jhund PS, Vazir A, Campbell R, McMurray JJ. Dynamic changes and prognostic value of pulmonary congestion by lung ultrasound in acute and chronic heart failure: a systematic review. Eur $\mathrm{J}$ Heart Fail 2017;19(9):1154-1163.

17. Dwyer KH, Merz AA, Lewis EF, Claggett BL, Crousillat DR, Lau ES, et al. Pulmonary congestion by lung ultrasound in ambulatory patients with heart failure with reduced or preserved ejection fraction and hypertension. J Card Fail 2018:24:219-226.

18. Gargani L, Pang PS, Frassi F, Miglioranza MH, Dini FL, Landi P, et al. Persistent pulmonary congestion before discharge predicts rehospitalization in heart failure: a lung ultrasound study. Cardiovasc Ultrasound. 2015;13:40.

19. Maw AM, Hassanin A, Ho PM, Mclnnes MDF, Moss A, Juarez-Colunga E, et al. Diagnostic accuracy of point-of-care lung ultrasonography and chest radiography in adults with symptoms suggestive of acute decom- pensated heart failure: a systematic review and meta-analysis. JAMA Netw Open. 2019; 2(3):e190703.

20. Gargani L, Frassi F, Soldati G, Tesorio P, Gheorghiade M, Picano E. Ultrasound lung comets for the differential diagnosis of acute cardiogenic dyspnoea: a comparison with natriuretic peptides. Eur J Heart Fail. 2008; 10:70-7.

21. Russell FM, Ehrman RR, Ferre R, Gargani L, Noble V,Rupp J, et al. Design and rationale of the $\mathrm{B}$-lines lung ultrasound guided emergency department management of acute heart failure (BLUSHED-AHF) pilot trial. Heart Lung. 2019:48;186-92.

22. ClinicalTrials.gov [Internet]. Bethesda (MD): National Library of Medicine (US). 2000 Feb 29. Identificador NCT03613779, Lung Ultrasound Guided Therapy in Heart Failure (CLUSTER-HF); 2018 Aug 3 [citado 2018 Nov 14]; 5 páginas]. Disponible en: https://clinicaltrials.gov/ct2/show/NCT03613779. 